\title{
Tolerability and outcomes of curative radiotherapy in patients aged 85 or more years
}

\author{
Patients of \\ advanced \\ age whose \\ performance \\ status is good \\ should still be \\ referred to be \\ assessed for \\ treatment
}

\section{Richard C Kho BMedSC, MBBS, FRANZCR \\ Mathias Bresse $\mathrm{BSC}, \mathrm{MSC}^{\mathrm{l}}$ \\ Jo Tedesco \\ MBIT}

Keen Hun Ta

MBBS, FRANZCR

David L Ball MBBS, MD, FRANZCR

Gillian M Duchesne MBChB, MD, FRANZCR.1.2

Helen Farrugia $\operatorname{BAppSc(HIM),~GradDip(BIT)}$

Wai Kuan Yip PhD, GradDipAppStats

Farshad Foroud MBBS, MPA, FRANZCR

I Peter MacCallum Cancer Centre Melbourne, VIC.

2 University of Melbourne Melbourne, VIC

3 Victorian Cancer Registry, Cancer Council Victoria, Melbourne, VIC

richard.khor@ petermac.org

doi: 10.5694/mjal4.00441 n developed nations, more than $50 \%$ of all cancer diagnoses are made in people aged over 65 years. ${ }^{1}$ It is well recognised that older patients are underrepresented in clinical trials. ${ }^{2-8}$ Older patients may be excluded from clinical trials because they tend to have high numbers of comorbid conditions, or because of a perception that they are unable to tolerate aggressive treatment., ${ }^{5,9}$ Populationlevel data show that older patients with many types of solid tumours are less likely to receive aggressive therapy, ${ }^{10-12}$ which may result in poorer outcomes. ${ }^{9}$

Our primary aim in this study was to document the tolerability of radiotherapy given with curative intent in patients aged 85 years and older.

\section{Methods}

This retrospective study was approved by the Peter MacCallum Cancer Centre ethics review committee (study 13/21).

\section{Participants}

Eligible patients included those aged 85 years and over who received $\mathrm{cu}$ rative radiotherapy at any Peter MacCallum Cancer Centre site between 1 January 2000 and 1 January 2010. Treatment was delivered in three metropolitan Melbourne sites, and one regional location (Bendigo). Patients must have received a dose of at least 25Gy to be included. Adjuvant radiotherapy or chemoradiation treatments were included. All presentations for non-metastatic cancers were included, except those for simple skin cancer treatments.

\section{Data collection}

For each patient, age, unadjusted Charlson comorbidity index (CCMI) score, Eastern Cooperative Oncology Group performance status (ECOG PS), and the type of malignancy were retrieved retrospectively. When calculating the CCMI score, the tumour

\section{Abstract}

Objectives: To assess the tolerability and survival outcome of curative radiotherapy in patients over the age of 85 years.

Design, setting, and participants: Retrospective analysis of all patients aged over 85 years who received radiotherapy as part of curative treatment for any cancer (excluding insignificant skin cancers) at the Peter MacCallum Cancer Centre between 1 January 2000 and 1 January 2010.

Main outcome measures: Poor treatment tolerability (defined as hospital admission during radiotherapy, treatment break, or early treatment cessation); predictors for poor treatment tolerability, overall survival and cancer-specific survival.

Results: 327 treatment courses met eligibility criteria. The median age of patients was 87 years. The most common treatment sites were pelvis (30\%), head and neck (25\%), and breast (18\%). The Eastern Cooperative Oncology Group performance status (ECOG PS) score was 0 or 1 for $70 \%$ of patients. Overall, $79 \%$ of patients completed the prescribed treatment without poor treatment tolerability, and $95 \%$ of patients completed all treatment. Only unfavourable ECOG PS score (odds ratio [OR], 1.80;

$P=0.005)$ and increasing age (OR, 1.18; $P=0.018)$ predicted poor treatment tolerability. ECOG PS score predicted overall survival (hazard ratio, 1.53; $P=0.001$ ).

Conclusion: Age should not be the sole discriminator in decisions to prescribe aggressive loco-regional radiotherapy. ECOG PS score predicts for treatment tolerability, and also overall survival. The risk of cancer death was higher than non-cancer death for more than 5 years after treatment.

diagnosis being treated was excluded from scoring. We proposed that increasing CCMI score might correlate with poor treatment tolerability. ${ }^{13}$

Radiation treatment details, including radiation dose and fractionation, the extent of treatment (primary, lymph node basins, or both), treatment body site and delivery of concurrent chemotherapy were available from electronic treatment records. The extent of treatment and treatment body site were grouped into categories that reflect the rigours of completing each course. If the radiation dose-fractionation was altered because of physician concerns about a patient's ability to tolerate the standard radiation treatment, this was termed "altered fractionation".

Poor treatment tolerability was defined as any instance of an interruption to or break from treatment, hospital admission from any cause, or early cessation of radiation treatment for any cause. The cause of each instance of poor treatment tolerability was assessed and assigned as either treatment-related or non-treatment-related.

Linkage with the Victorian Cancer Registry (VCR) facilitated high fidelity national-level mortality information. Overall survival was measured for all patients included in the VCR, and was measured from the first radiotherapy fraction. A closeout date of 31 December 2011 was used, as VCR survival data are complete up until this date. All patients without a recorded death date before the closeout date were considered alive on that date. All patients were censored at the closeout date.

\section{Statistical analysis}

The statistical package $R$, version 3.0 (R Foundation for Statistical Computing; http://www.R-project. org) was used for statistical analysis. Quantitative variables were described using summary statistics. Poor treatment tolerability was described using counts and percentages, with 95\% confidence intervals calculated using exact methods. Logistic regression 
Univariate and multivariate analyses of factors associated with poor tolerability of radiotherapy in 325 patients aged over 85 years

\begin{tabular}{|c|c|c|c|c|c|c|}
\hline \multirow[b]{2}{*}{ Variable } & \multirow{2}{*}{$\begin{array}{l}\text { Number of } \\
\text { patients }\end{array}$} & \multirow{2}{*}{$\begin{array}{c}\text { Poor treatment } \\
\text { tolerability }\end{array}$} & \multicolumn{2}{|c|}{ Univariate analysis } & \multicolumn{2}{|c|}{ Multivariate analysis } \\
\hline & & & Odds ratio $(95 \% \mathrm{Cl})$ & $P$ & Odds ratio $(95 \% \mathrm{Cl})$ & $P$ \\
\hline Extent of radiation treatment & & & & 0.092 & & 0.145 \\
\hline Primary tumour plus lymph node basins & 131 & $27 \%$ & 1 & & 1 & \\
\hline Primary tumour only & 174 & $18 \%$ & $0.57(0.33-0.99)$ & & $0.85(0.43-1.69)$ & \\
\hline Lymph node basins only & 21 & $14 \%$ & $0.44(0.10-1.40)$ & & $0.24(0.01-1.35)$ & \\
\hline Concurrent chest x-ray & & & & 0.379 & & 0.920 \\
\hline No & 251 & $19 \%$ & 1 & & 1 & \\
\hline Yes & 71 & $24 \%$ & $1.33(0.70-2.46)$ & & $1.03(0.46-2.23)$ & \\
\hline CCMI score (ordinal) & & & $1.03(0.80-1.30)$ & 0.806 & $1.05(0.78-1.38)$ & 0.605 \\
\hline ECOG performance status score (ordinal) & & & $1.73(1.23-2.44)$ & 0.001 & $1.80(1.23-2.69)$ & 0.005 \\
\hline ECOG performance status score (grouped) & & & & $<0.001$ & & \\
\hline O or 1 & 229 & $16 \%$ & 1 & & & \\
\hline 2 or 3 & 52 & $44 \%$ & $4.3(2.21-8.19)$ & & & \\
\hline Age (continuous) & & & $1.10(0.99-1.22)$ & 0.088 & $1.18(1.04-1.34)$ & 0.013 \\
\hline Altered fractionation & & & & 0.784 & & 0.113 \\
\hline No & 236 & $20 \%$ & 1 & & 1 & \\
\hline Yes & 81 & $19 \%$ & $0.92(0.47-1.71)$ & & $0.53(0.23-1.15)$ & \\
\hline
\end{tabular}

CCMI = Charlson comorbidity index. ECOG = Eastern Cooperative Oncology Group.

was used to evaluate the impact of possible prognostic factors of poor tolerability. Overall survival and cancer-specific survival were estimated using Kaplan-Meier methods with $95 \%$ confidence intervals. As patients with more than one cancer diagnosis were included in the study, cancerspecific survival was measured in respect to the cancer being treated during the study period. Cox proportional hazard models were used to test whether ECOG and CCMI were associated with overall survival. ECOG and CCMI were treated as ordinal variables. Cumulative incidence curves for cause of death were provided assuming competing risks. Median follow-up was calculated using the reverse Kaplan-Meier method with $95 \%$ confidence interval.

\section{Results}

Between 1 January 2000 and 1 January 2010 a total of 327 radiotherapy courses were delivered to 325 patients who met our inclusion criteria. In two courses, information regarding treatment breaks could not be retrieved. The baseline patient and treatment characteristics for the study population are available online (Appendix 1). The most common sites treated were pelvis (30\%), head and neck (25\%), breast (18\%) and thorax (13\%). The 10 most common diagnosis groups and prescribed total radiation dose and fractions are available in Appendix 2.

\section{Tolerability analysis}

Poor treatment tolerability was observed in 70 patients (21\%; estimated 95\% CI, 17\%-26\%). Only 16 patients (5\%) ceased treatment prematurely. The characteristics of treatment tolerability are described in Appendix 3. Univariate analysis of the predictors of poor treatment tolerability showed that only unfavourable ECOG PS scores before treatment commencement predicted poor treatment tolerability (odds ratio [OR], 1.73; $P=0.001$ ). Rates of poor treatment tolerability for patients with ECOG PS scores (higher scores indicate poorer performance) of $0,1,2$ and 3 were $16 \%$
(16/97), 15\% (20/132), 49\% (18/37) and $33 \%(5 / 15)$, respectively. We also analysed ECOG PS score as a categorical variable, reflecting clinical practice, of patients with "good" PS (ECOG score 0-1) and "poor" PS (ECOG scores 2-3). On multivariate analysis, ECOG PS score remained statistically significant (OR, 1.80; $P=0.005$; 95\% CI, 1.23-2.69), and increasing age was found to be a statistically significant predictor of poorer tolerability (OR 1.18; $P=0.018 ; 95 \%$ CI, 1.04-1.34). Because of the small number of patients with a ECOG PS score of 3 , the multivariate analysis was repeated with ECOG PS scores grouped as described above, and again only ECOG PS remained significant (data not shown). The Box shows the results of the univariate and multivariate analyses.

\section{Survival analysis}

Registry-level survival data were available for $97 \%$ of the patient cohort (314/325). Survival estimates for $1,3,5$ and 8 years were $81.2 \%, 52.7 \%$, $38.1 \%$ and $20.2 \%$, respectively. The 
overall survival curve can be viewed in Appendix 4. Cancer-specific survival estimates at 1, 3, 5 and 8 years were $87 \%, 66 \%, 56 \%$ and $44 \%$, respectively. The median follow-up period was 6 years (95\% CI, 5.1-6.6 years) and median survival was 3.3 years (95\% CI, 2.7-4.1 years).

Cause of death was available from the VCR in all but two cases. The competing risks analysis (312 patients; Appendix 5) showed that the risk of cancer-related death exceeded that of death from other causes.

Five-year overall survival decreased with unfavourable ECOG PS (overall survival estimates with PS of 0 , 1,2 and 3 were $53 \%, 33 \%, 22 \%$ and $8 \%$, respectively). Increasing ordinal ECOG PS scores correlated significantly with poorer overall survival (hazard ratio [HR], 1.53; $P=0.001$; 95\% CI, 1.29-1.81). Figures describing overall survival curves by ECOG PS score are available in Appendix 6. Increasing CCMI was also associated with poorer survival outcome (HR, 1.16; $P=0.023$; 95\% CI, 1.02-1.31).

\section{Discussion}

In this large cohort study of elderly patients treated with curative radiotherapy, our primary objective was to assess the tolerability of highdose radiotherapy. Our data show that, with judicious patient selection, patients over the age of 85 years can tolerate aggressive radiotherapy regimens. The treatment completion rate of $95 \%$ was higher than in other cohort studies with less stringent entry criteria. ${ }^{14-16}$ The limited number of existing publications often included low numbers of elderly patients ${ }^{17}$ or were small, ${ }^{14-16}$ included palliative treatments, ${ }^{14-16}$ or were limited by short follow-up time. ${ }^{16}$

The outcomes of our study may be affected by selection bias and inclusion of the "well" old; $70 \%$ of the patients in our study had an ECOG PS score of 0 or 1 and $72 \%$ had a CCMI score of 0 . In contrast, a study of comorbid conditions in patients with breast cancer who were over the age of 80 years found that only $35 \%$ of patients were free of common comorbid conditions. ${ }^{9}$ Such selection bias is difficult to avoid as it may begin before patients are referred by treating doctors to be considered for radiotherapy. That is, treating doctors may not refer frailer patients on the presumption they will not tolerate treatment, when, in fact, they may have proceeded through radiotherapy without problems. Referring only relatively fitter patients who are more likely to tolerate side effects may create undertreatment biases.

In our cohort of patients, $22 \%$ were prescribed alternative dose-fractionation radiotherapy courses, which are easier for patients to tolerate because there are a smaller number of fractions. We included these patients, as excluding a poor prognosis group from analysis would have introduced further selection bias. On balance, altered fractionation was included in the multivariate analysis of predictors of poor treatment tolerability, and was found not to predict poor tolerability. As frail patients are often offered altered fractionation regimens that are easier to tolerate, treatment tolerability was preserved.

ECOG PS is related to the concept of geriatric "frailty", which defines the progressive age-related loss of selfmanagement skills. Frailty is also associated with poorer outcome after other health interventions. ${ }^{18}$ Among patients for whom radical treatment is contemplated, we found that ECOG PS score predicts both tolerability and survival. ECOG PS thus remains a useful predictive factor despite its lack of granularity. Ideally, a formal comprehensive geriatric assessment $(\mathrm{CGA})^{19}$ should be used as pre-treatment assessment. The CGA has been shown to predict survival ${ }^{20}$ and the incidence of side effects ${ }^{20,21}$ and, most importantly, to change clinical treatment decisions. ${ }^{22}$

This study reinforces the findings of the limited literature describing the favourable tolerability of radiotherapy in very elderly patients. It is perhaps incorrectly perceived that a high rate of natural attrition in this age group lessens the need for longterm cancer control. We challenge this assumption; the median survival in our study was 3.4 years, which is shorter than the life expectancy for an 87-year-old Australian (6.2 years for men and 7.2 years for women). ${ }^{23}$

In summary, loco-regional radiotherapy is well tolerated by older patients with cancer. Patients of advanced age whose performance status is good should still be referred to be assessed for treatment.

Competing interests: No relevant disclosures. 
1 Emanuel EJ, Emanuel LL. The economics of dying. The illusion of cost savings at the end of life. NEngl J Med 1994; 330: 540-544.

2 Balducci L, Beghe C. Cancer and age in the USA. Crit Rev Oncol Hematol 2001; 37: 137-145.

3 Benson AB 3rd, Pregler JP, Bean JA, et al. Oncologists' reluctance to accrue patients onto clinical trials: an Illinois Cancer Center study. J Clin Oncol 1991; 9: 2067-2075.

4 Hutchins LF, Unger JM, Crowley JJ, et al. Underrepresentation of patients 65 years of age or older in cancertreatment trials. N Engl J Med 1999; 341: 2061-2067.

5 Kemeny MM, Peterson BL, Kornblith $A B$, et al. Barriers to clinical trial participation by older women with breast cancer. J Clin Oncol 2003; 21: 2268-2275.

6 Lewis JH, Kilgore ML, Goldman DP, et al. Participation of patients 65 years of age or older in cancer clinical trials. J Clin Oncol 2003; 21: 1383-1389.

7 Trimble EL, Carter CL, Cain D, et al. Representation of older patients in cancer treatment trials. Cancer 1994; 74 (7 Suppl): 2208-2214.

8 Yee KW, Pater JL, Pho L, et al. Enrollment of older patients in cancer treatment trials in Canada: why is age a barrier? J Clin Oncol 2003; 21: 1618-1623.

9 Louwman WJ, Janssen-Heijnen ML, Houterman S, et al. Less extensive treatment and inferior prognosis for breast cancer patient with comorbidity: a population-based study. Eur J Cancer 2005; 41: 779-785.

10 Fedeli U, Fedewa SA, Ward EM. Treatment of muscle invasive bladder cancer: evidence from the National Cancer Database, 2003 to 2007. J Urol 2011; 185: 72-78.

11 D’Amico AV, Whittington R, Malkowicz SB, et al. Biochemical outcome after radical prostatectomy, external beam radiation therapy, or interstitial radiation therapy for clinically localized prostate cancer. JAMA 1998; 280: 969-974.

12 Schonberg MA, Marcantonio ER, Li D, et al. Breast cancer among the oldest old: tumor characteristics, treatment choices, and survival. J Clin Oncol 2010; 28: 2038-2045.

13 Charlson M, Szatrowski TP, Peterson J, Gold J. Validation of a combined comorbidity index. J Clin Epidemiol 1994; 47: 1245-1251.

14 Oguchi M, Ikeda $\mathrm{H}$, Watanabe $\mathrm{T}$, et al. Experiences of 23 patients $>$ or $=90$ years of age treated with radiation therapy. Int J Radiat Oncol Biol Phys 1998; 41: 407-413.

15 Ikeda H, Ishikura S, Oguchi M, et al. Analysis of 57 nonagenarian cancer patients treated by radical radiotherapy: a survey of eight institutions. Jpn J Clin Oncol 1999; 29: 378-381.

16 Thompson A, Cone R, Gao H, et al. Is advanced age a barrier to effective cancer treatment? The experience of nonagenarians receiving radiation therapy. Asia Pac J Clin Oncol 2012; 8: 255-259.
17 Pignon T, Gregor A, Schaake Koning C, et al. Age has no impact on acute and late toxicity of curative thoracic radiotherapy. Radiother Oncol 1998; 46: 239-248.

18 Schuurmans $\mathrm{H}$, Steverink $\mathrm{N}$, Lindenberg $\mathrm{S}$, et al. Old or frail: what tells us more? J Gerontol A Biol Sci Med Sci 2004; 59: M962-M965.

19 Extermann M, Hurria A. Comprehensive geriatric assessment for older patients with cancer. J Clin Oncol 2007; 25: 1824-1831.

20 Hamaker ME, Prins MC, Stauder R. The relevance of a geriatric assessment for elderly patients with a haematological malignancy - a systematic review. Leuk Res 2014; 38: 275-283.

21 Extermann M, Boler I, Reich RR, et al. Predicting the risk of chemotherapy toxicity in older patients: the Chemotherapy Risk Assessment Scale for High-Age Patients (CRASH) score. Cancer 2012; 118: 3377-3386.

22 Kenis C, Bron D, Libert Y, et al. Relevance of a systematic geriatric screening and assessment in older patients with cancer: results of a prospective multicentric study. Ann Oncol 2013; 24: 1306-1312.

23 Australian Bureau of Statistics. Life tables, states, territories and Australia, 2009-2011. Canberra: ABS, 2012. (ABS Cat. No. 3302.0.55.001.) http://www. abs.gov.au/AUSSTATS/abs@.nsf/allpri marymainfeatures/8B2EC768599A2B5 5CA257C1B000D755A?opendocument (accessed Nov 2013). 